\title{
Nivolumab-induced lichenoid granulomatous stomatitis in a patient with advanced melanoma: A case report
}

\author{
P. GOUVERIS ${ }^{1}$, E. A. GEORGAKOPOULOU ${ }^{2}$, A. GRIGORAKI ${ }^{3}$, D.N. ZOUKI ${ }^{1}$, V.E. KARDARA ${ }^{1}$, \\ S. IOANNOU ${ }^{1}$, D. TRYFONOPOULOS ${ }^{1}$, S. DEMIRI $^{1}$ and I. GKOUVERIS ${ }^{4}$ \\ ${ }^{1}$ Second Department of Medical Oncology, Agios Savvas Cancer Hospital, 11522 Athens; \\ ${ }^{2}$ Laboratory of Histology-Embryology Molecular Carcinogenesis Group; ${ }^{3}$ Department of Pathology, \\ School of Medicine, National and Kapodistrian University of Athens; ${ }^{4}$ Department of Oral and \\ Maxillofacial Pathology and Medicine, School of Dentistry, National and \\ Kapodistrian University of Athens, 11527 Athens, Greece
}

Received November 15, 2021; Accepted January 18, 2022

DOI: $10.3892 / \mathrm{mco} .2022 .2512$

\begin{abstract}
Lichenoid granulomatous reactions (LGR) are granulomatous inflammations of the skin and oral mucosa, also sharing features of lichenoid lesions. Thus, the present study refers to lichenoid granulomatous dermatitis (LGD) and lichenoid granulomatous stomatitis (LGS). LGR is a condition that can be triggered by drugs, diseases or environmental causes. In the present case study, anti-PD1 (nivolumab) medication had a detrimental effect on the oral mucosa, which clinicaly and histologicaly proved to be LGS. Checkpoint inhibitors consitute a cornerstone in the current treatment of several types of cancer, of which cutaneous melanoma is the best example. Oral lichenoid responses following anti-PD-1 therapy have been recorded in few case reports and small case series. To the best of our knowledhe, this is the first case of LGS being reported as a side effect of immune checkpoint inhibitor treatment.
\end{abstract}

\section{Introduction}

Immune checkpoint inhibitors represent novel, promising and effective therapeutic approaches for many cancers. However, such therapies are frequently followed by

Correspondence to: Dr Dionysia N. Zouki, Second Department of Medical Oncology, Agios Savvas Cancer Hospital, 171 Alexandras Avenue, 11522 Athens, Greece

E-mail: dzouki@med.uoa.gr

Abbreviations: LGR, lichenoid granulomatous reactions; LGD, lichenoid granulomatous dermatitis; LGS, lichenoid granulomatous stomatitis; PD-1, programmed cell death protein 1; PD-L1, programmed cell death ligand 1; AFB, acid-fast bacilli; GMS, grocott methenamine-silver; PAS, periodic acid-schiff; OLR, oral lichenoid reactions

Key words: immune-related adverse events, lichenoid granulomatous stomatitis, nivolumab, melanoma immune-related adverse events that may affect variable systems and organs including colitis, dermatitis, hepatitis, pneumonitis and thyroiditis. Most of them occur within the first few months of immunotherapy. Oncologists may often need collaboration with physicians from other specialties, like gastroenterologists, dermatologists, pulmonologists, endocrinologists, in cases of high grade or persistent toxicity.

Oral cavity may also be affected by drug related toxicity $(1,2)$. Clinician often describe oral toxicities with general terminologies like mucositis or stomatitis (2). Stomatitis has been recorded as a potential side effect in phase 3 trials with checkpoint inhibitors like nivolumab $(1,3)$. However, in these great randomized trials there was no search for the exact type of stomatitis, perhaps due to the fact that there were no serious adverse events regarding oral cavity (4). A few cases of oral lichenoid reactions have been described in the literature as potential 'stomatitis' lesions following anti-PD-1 therapy (5-9). These anti-PD1-related toxicities were histologically consistent with oral lichenoid reactions, however tended to display a greater histiocytic infiltrate compared to those described in nondrug-induced lichenoid responses (8).

Moreover, specific etiologic factors like foreign materials, infective organisms, and immunologic agents are responsible for the development of chronic granulomatous inflammation in oral cavity (10). Interestingly, anti-PD1 treatment has also been associated with development of certain granulomatous disease including sarcoid-like granulomatous reaction, granuloma annulare and granulomatous inflammation of the pleura (11-13).

Furthermore, lichenoid granulomatous reactions (LGR), observed in skin and oral mucosa, and reported as lichenoid granulomatous dermatitis (LGD) and lichenoid granulomatous stomatitis (LGS), respectively, represent mixed entities that display a combination of granulomatous inflammation with a histologic type consistent with lichenoid lesions (14). LGR may evolve as a result of certain medications or other etiologic factors like common pathogens or foreign materials (15-18). 
This study presents for the first time a case of LGR in the oral mucosa, as a side-effect of immune checkpoint inhibitor treatment and it refers to a patient who received nivolumab. Nivolumab is a known human IgG4 monoclonal antibody that blocks PD-1 and releases T-cell activated immunity against cancer cells.

\section{Case report}

A 67-year-old female patient was diagnosed 2 years ago with cutaneous melanoma of inner right thigh, Clark III, Breslow $3.5 \mathrm{~mm}$, mitotic rate $15 / \mathrm{mm}^{2}$, with ulceration, pT3bNOM0, stage IIB. Her medical history was free, with absence of allergies and autoimmune disorders. Following initial diagnosis, the patient underwent wide excision and sentinel inguinal lymph node biopsy and was set under regular observation. After one and a half year, she presented with a suspicious nodule of right groin, at the area of the sentinel lymph node biopsy scar. There was a surgical excision and the histological examination revealed subcutaneous melanoma metastasis. The patient started adjuvant immunotherapy with nivolumab every two weeks. One week after the first cycle, she complained of intense mouth pain. Nevertheless, the patient received the second cycle of nivolumab and was further evaluated by the oncology team. Stomatitis grade 2 was initially diagnosed, showing no involvement of face or body skin area. Local antifungal treatment was prescribed, but stomatitis persisted and got worse. The patient had severe pain and eating discomfort. Consequently, she was referred to an Oral Medicine specialist who observed several atrophic, erythematous areas with central ulcerations of varying degrees mostly located bilaterally at the sides of the tongue and at the mucosa of both upper and lower lip. Moreover, at the periphery of the atrophic regions, a mild white radiating striae was hardly noticed (Fig. 1).

Biopsies were taken from the mucosa of the upper lip for histological examination and definite diagnosis. Histologic evaluation revealed mild local hyperkeratosis, mild spongiosis, formation of colloid bodies, degeneration of the basal layer and a band- like lymphocyte infiltration, also containing numbers of histiocytes, neutrophils and eosinophils in the superficial lamina propria (Fig. 2A). Deeper in the corium, increased desmoplastic reaction was observed as well as aggregates of granulomatous inflammation consisting of lymphocytes and histiocytes (Fig. 2B), clustering around scattered vessels leading to mild vascular occlusions (Fig. 2C). Further immunohistochemical staining demonstrated CD68-positive cells and prevalence of CD4 vs. CD8 lymphocytes. Special staining for microorganisms including acid-fast bacilli (AFB), grocott methenamine-silver (GMS), and periodic acid-Schiff (PAS) was also conducted. Positivity was noticed only in GMS staining representing numbers of bacteria inside the corium and phagocytosised by histiocytes (Fig. 3).

Following clinical and histopathologic evaluation, a final diagnosis of LGS was given. The patient was treated with topical corticosteroids in addition to doxycycline $40 \mathrm{mg}$ twice a day. After 2 weeks of treatment lesions totally resolved. However, the patient was so distressed by the whole situation that she refused to go on with immunotherapy. There was a permanent discontinuation of nivolumab treatment. Eight months after treatment discontinuation, the patient is free from malignant melanoma recurrence.

\section{Discussion}

Patients treated with immune checkpoint inhibitors targeting PD-1 or PD-L1, frequently present specific dermatologic toxicities including cutaneous lichenoid reactions $(8,9,19,20)$. Interestingly, oral mucous involvement may often occur while oral lichenoid reactions (OLR) have only anecdotally been reported $(8,9,20)$. Indeed, 7 cases of OLR following anti-PD-1 therapy have already been described in the English literature (5-9). The majority of patients suffered from multiple erosions or ulcers and clinically presented stomatitis of variable grading. Histopathological examination of the lesions was compatible with oral lichen planus, demonstrating epithelial necrosis and spongiosis with a dense band-like layer of inflammatory infiltrate within the upper mucosa, consisting of lymphocytes and histiocytes (5-9). Further examinations like direct immunofluorescence excluded other related mucocutaneous entities in all cases (5-9). As a reminding notice, oral lichen planus is characterized by six clinical variants including reticular, papular, plaque-like, erosive, atrophic and bullous types. Nevertheless, 6 of the 7 aforementioned cases presented with multiple painful erosions and ulcers mainly located in soft/hard palate as well as lip and buccal mucosa (5-7,9). Subsequent rash treatment included topical or systemic corticosteroid administration depending on existence of adjacent cutaneous lesions, while OLR presence variably affected normal antitumor therapy continuation (5-9).

Although our case appeared clinically as an oral lichenoid reaction, it was histologically accompanied by granulomatous infiltration. Therefore, it was finally diagnosed in the context of a different morphologic spectrum of lichenoid reaction which is named lichenoid granulomatous reaction. The later could be divided into LGD and LGS (14). Treatment with nivolumab is the cause that triggered the lichenoid granulomatous reaction in our patient, as the first symptoms of stomatitis occurred one week after the first cycle of therapy, and diagnosis was established a few weeks later, with symptoms persisting and getting worse despite the local antifungal treatment. Fig. 4 represents the patient's course from melanoma diagnosis till the subsequent initiation of nivolumab as well as the onset and resolution of stomatitis. This is the first LGS case ever reported as a side effect of an immune checkpoint inhibitor, while only one nivolumab-induced LGD case has been also described in 2018 (21). Gonzalez et al (16) initially presented this entity in 1986 while Magro and Crowson, in 2000, examined it in 40 patients (17). Lichenoid granulomatous reaction shows a histologic profile of band-like lymphocytic infiltrates in close opposition to the epidermis or mucosa and clusters of histiocytic or granuloma formation in the dermis or corium. In addition, Magro and Crowson proposed 5 histologic patterns according to histiocytic infiltration that included: i) superficially disposed loose small histiocytic aggregates amidst a band-like lymphocytic infiltrate; ii) small epithelioid granulomata within and subjacent to the areas of band-like lymphocytic infiltration; iii) an interstitial array between collagen fibers reminiscent of interstitial granuloma annulare; iv) scattered singly disposed Langhans and/or foreign 
A

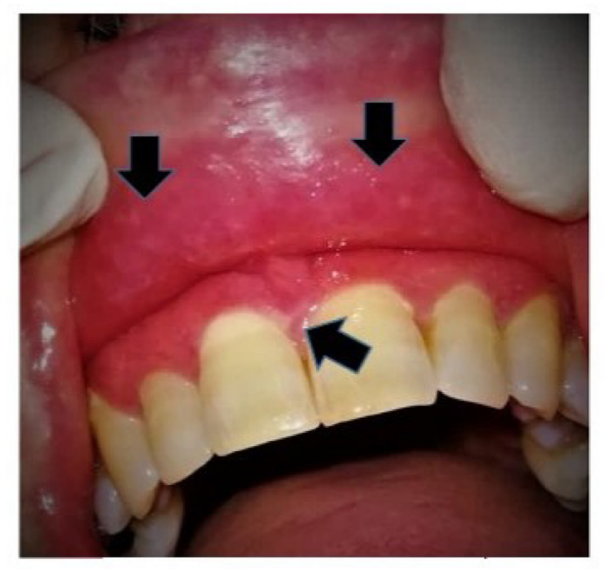

C

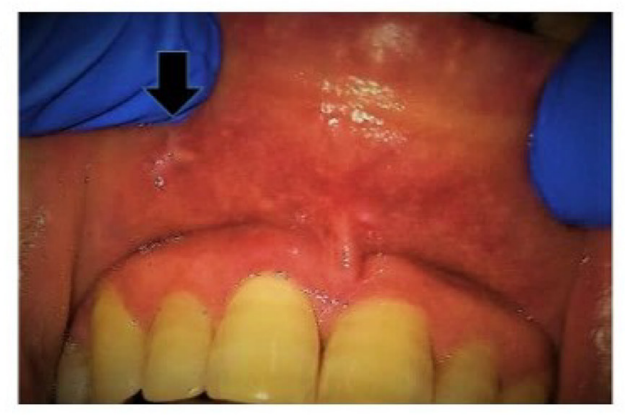

B

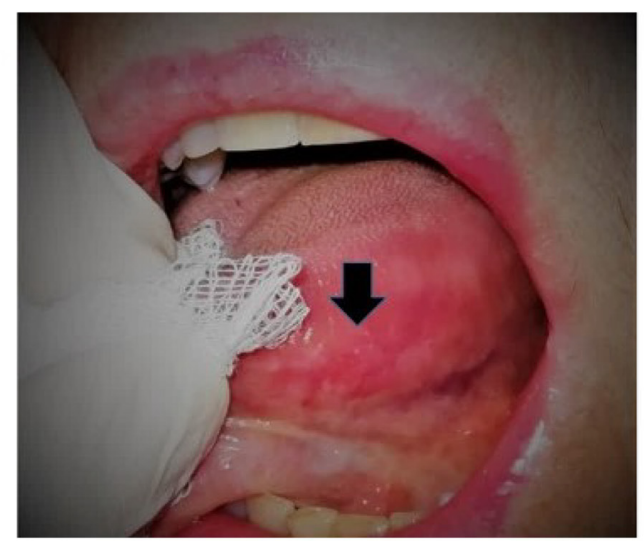

D

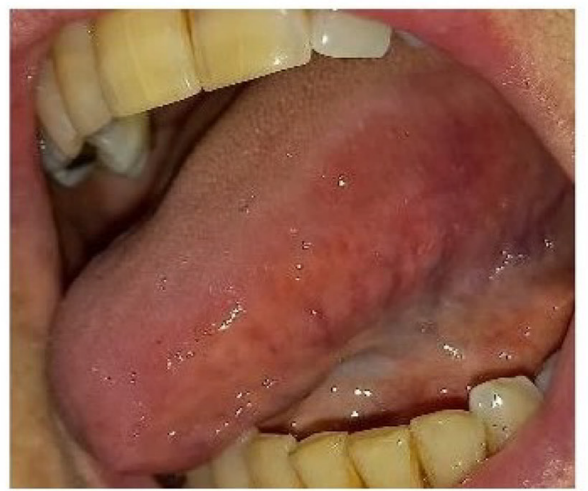

Figure 1. Representative images of oral mucosal lesions. Indicative atrophic, erythematous areas with central ulcerations of varying degrees at the left side of the tongue and at the mucosa of both upper and lower lip. (A) Lip lesions erythema and papules on the mucosa of the lips and gingiva (arrows). These lesions had not responded to topical clobetasol and a biopsy was performed. (B) Tongue lesions erythema and very mild erosion (arrow). (C) Lip lesions improved 7 days after the beginning of doxycycline $40 \mathrm{mg}$ OD and dexamethasone solution $\mathrm{BD}$ (note the site of the biopsy arrow). (D) Tongue lesions healed 7 days after the beginning of doxycycline $40 \mathrm{mg}$ OD and dexamethasone solution $\mathrm{BD}$. OD, once a day; BD, twice a day.
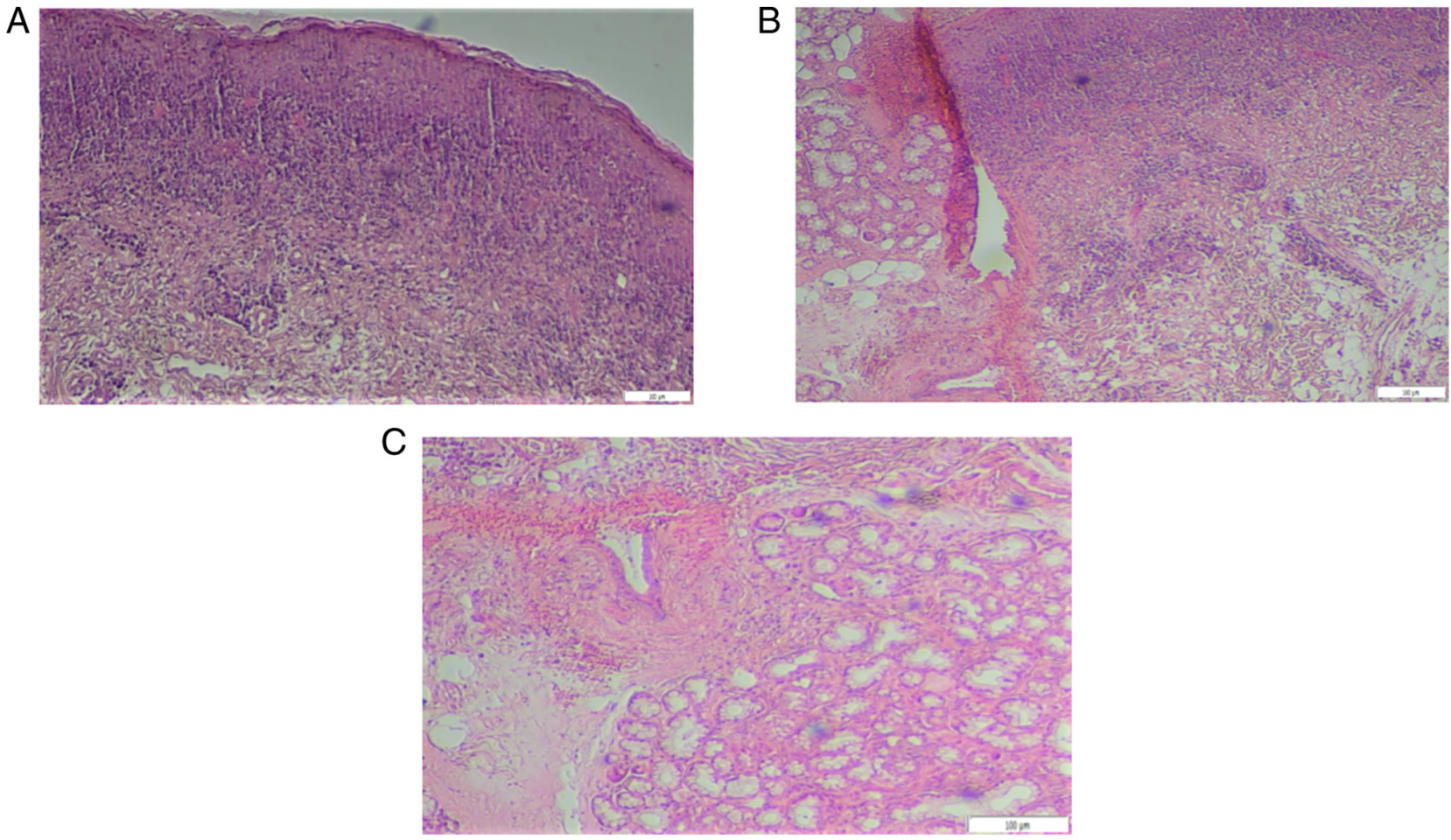

Figure 2. Histological examination. (A) Mild hyperkeratosis of the stratified squamous epithelium and dense inflammatory lymphocytic and mast cell infiltrates with diffuse subepithelial distribution and disruption of normal architecture of the epithelium-dermis margin. (B) Dense inflammatory lymphocytic subepithelial infiltrates converging to form granulomatous like structures. (C) Deep vascular branch with perivascular granulomatous infiltrates. 

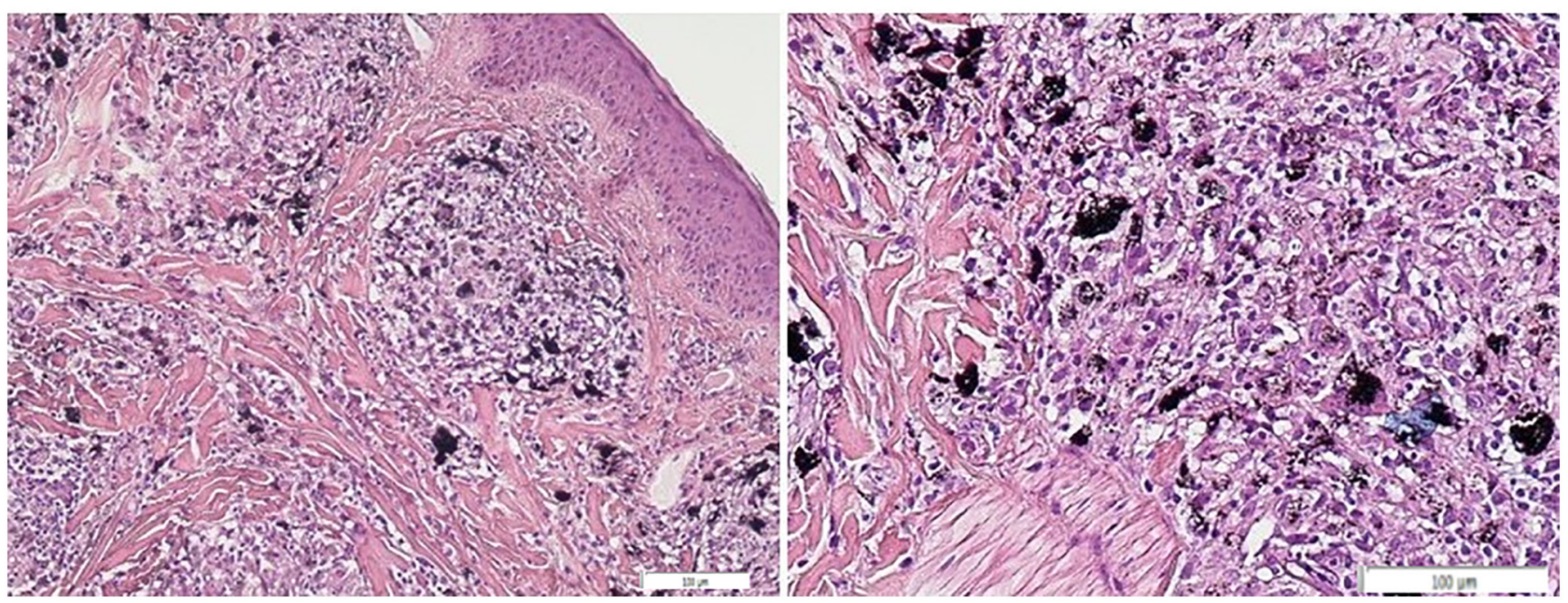

Figure 3. GMS staining. Indicative areas of GMS staining representing numbers of bacteria inside the corium which are also phagocytosed by histiocytes (magnification, x20 left; x40 right). GMS, Grocott methenamine-silver.

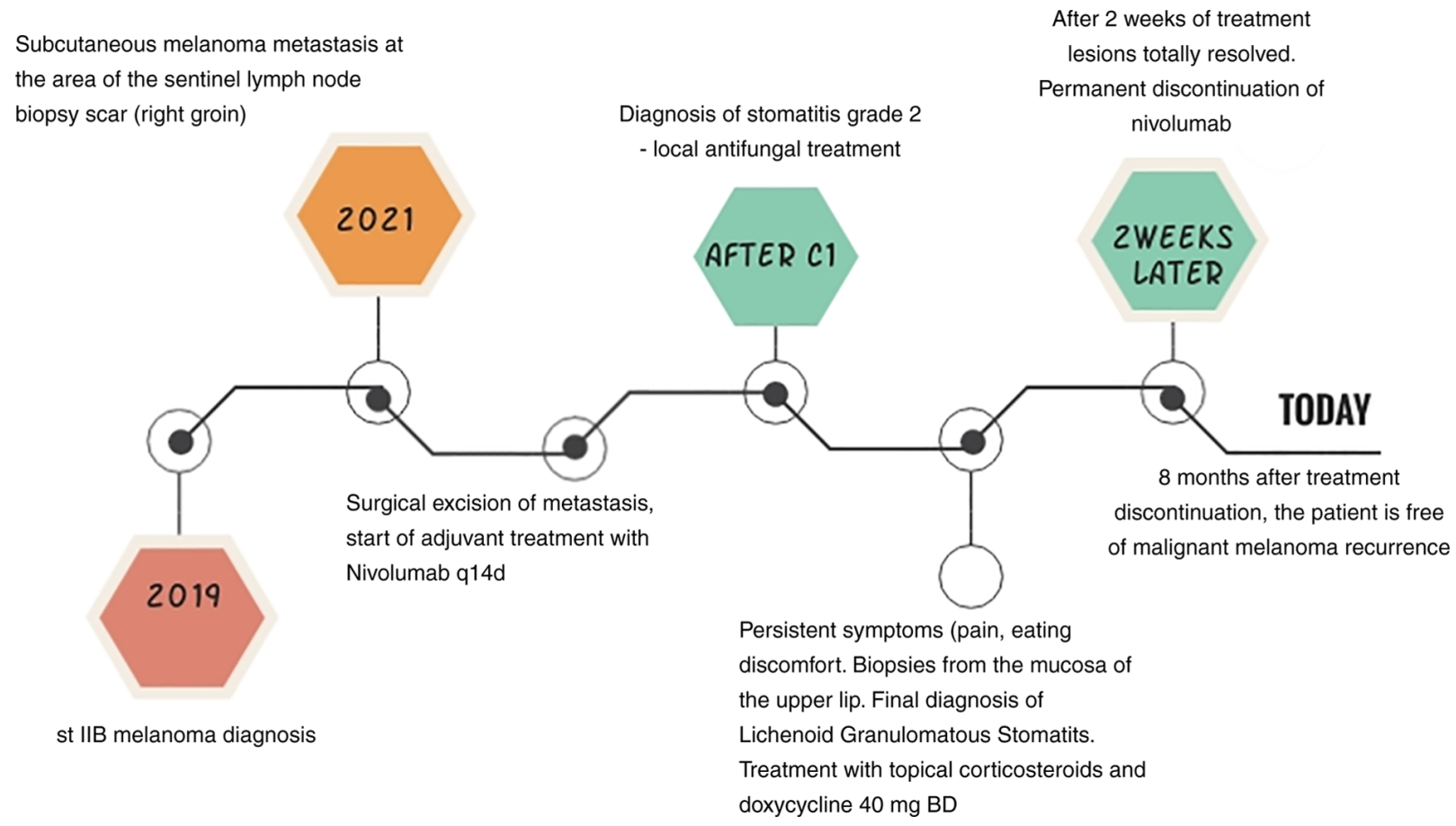

Figure 4. Patient's timeline from initial melanoma diagnosis till the subsequent initiation of nivolumab therapy as well as the onset and resolution of stomatitis. GMS, Grocott methenamine-silver.

body type giant cells; and v) granulomatous vasculitis (17). Following this classification, the present case may be classified as type II LGS presenting band-like lymphocytic infiltration with loose histiocyte aggregates, showing also signs of initiating perivasculitis.

LGS incidence shows a female predilection (64\%) whereas the most common sites of lesion occurrence include gingiva, buccal mucosa, vestibule, tongue, lip and palate. Initial clinical diagnosis may refer to lichen planus, vesiculobullous disease, leukoplakia, dysplasia, carcinoma in situ and allergy (14). Furthermore, both LGD and LGS may evolve as a result of drug eruptions $(14,18,22)$, cutaneous T-cell lymphoma, immune response to preceding viral infections or active infections, hepatobiliary disorders and rheumatoid arthritis (1). Regarding nivolumab involvement, the specific mechanism that drives anti-PD-1 mediated drug eruptions is still obscure. However, the role of PD-1 pathway in the induction and maintenance of immune tolerance has already been identified (7). On the other hand, anti-PD-1 treatment enhances T-cell activation by inhibiting the PD-1 suppressive effect on $\mathrm{T}$ cells, thus provoking anti-tumor activity in certain cancers (23). In addition, anti-PD-1 therapy enforces antigen recognition and T-cell proliferation in lymph nodes that induces systemic cytotoxic T-cell effects that finally involve both normal tissues and cancer cells $(23,24)$. Nonetheless, it has already been reported that PD-L1/PD-1 pathways appear to be compromised in oral 
lichen planus (25) and therefore anti-PD-1 administration may further enhance lichenoid reactions. More recent studies imply that nivolumab treatment is involved in certain granulomatous eruptions including sarcoid-like granulomatous reaction, granuloma annulare and granulomatous inflammation of the pleura (11-13). In current case report, local bacterial infection or excessive immune response against local oral microbiome may be the cause of granuloma formation in addition to the nivolumab-induced T-cell proliferation that drives the lichenoid reaction. In agreement, Magro and Crowson also report that lichenoid and granulomatous infiltrates could occur as a result of active infection, or idiopathic response to nonviable microbial antigen like microbial proteins from viruses, mycobacteria, treponema and streptococci (17).

There are no preventative drugs for stomatitis. Close follow up of a patient with drug-induced stomatitis is essential. The patient must be referred to an Oral Medicine specialist when the problem persists. Topical steroid administration is the most common LGS therapeutic approach (14), while in present case systemic doxycycline was also given due to the microbial involvement. Doxycycline also has anti-inflammatory action in granulomatous inflammation (25).

In conclusion, clinicians should be aware that LGD and LGS are two rare but severe muco-cutaneous lesions that may occur in patients under anti-PD-1 medication. Since this discomfort could lead to therapy discontinuation, early diagnosis and proper management would be of great benefit.

\section{Acknowledgements}

Not applicable.

\section{Funding}

No funding was received.

\section{Availability of data and materials}

All data generated or analyzed during this study are included in this published article.

\section{Authors' contributions}

PG and IG conceptualized the study. PG, EAG and AG curated the data. DNZ and PG designed the methodology and interpretated the data. PG, EAG, VEK and SI wrote the original draft and obtained/designed the figures. DT, IG, SD made substantial contribution to the acquisition of data, reviewed and edited this manuscript and confirm the authenticity of all the raw data. All authors have read and approved the final manuscript and agree to be accountable for all aspects of the work in ensuring that questions related to the accuracy or integrity of any part of the work are appropriately investigated and resolved.

\section{Ethics approval and consent to participate}

Not applicable. This case report was conducted in accordance with the 1964 Helsinki Declaration and its later amendments or comparable ethical standards. Written informed consent was obtained from the patient.

\section{Patient consent for publication}

Written informed consent was obtained from the patient for publication of this case report and any accompanying images.

\section{Competing interests}

The authors declare that they have no competing interests.

\section{Authors' information}

The ORCID iD for Dr Dionysia N. Zouki is 0000-00020623-1323.

\section{References}

1. Mishra S, Bajoria AA, Parihar AS, Kochhar AS, Bhasin R and Bharadwaj A: Evaluation of lichenoid granulomatous stomatitis cases: A Retrospective Study. J Nat Rem 21: 55-59, 2020.

2. Vigarios E, Epstein JB and Sibaud V: Oral mucosal changes induced by anticancer targeted therapies and immune checkpoint inhibitors. Support Care Cancer 25: 1713-1739, 2017.

3. Motzer RJ, Escudier B, George S, Hammers HJ, Srinivas S, Tykodi SS, Sosman JA, Plimack ER, Procopio G, McDermott DF, et al: Nivolumab versus everolimus in patients with advanced renal cell carcinoma: Updated results with long-term follow-up of the randomized, open-label, phase 3 CheckMate 025 trial. Cancer 126: 4156-4167, 2020.

4. Dika E, Lambertini M, Gouveia B, Mussi M, Marcelli E, Campione E, Gurioli C, Melotti B, Alessandrini A and Ribero S: Oral manifestations in melanoma patients treated with target or immunomodulatory therapies. J Clin Med 10: 1283, 2021.

5. Enomoto Y, Nakatani H, Kondo S, Kasai T and Tsuchiya Y: Drug-induced oral lichenoid reaction during nivolumab therapy. Int J Oral Maxillofac Surg 48: 488-491, 2019.

6. Namiki T, Hanafusa T, Ueno M, Miura K and Yokozeki H: Severe oral ulcers associated with nivolumab treatment. JAMA Dermatol 153: 235-237, 2017.

7. Obara K, Masuzawa M and Amoh Y: Oral lichenoid reaction showing multiple ulcers associated with anti-programmed death cell receptor-1 treatment: A report of two cases and published work review. J Dermatol 45: 587-591, 2018.

8. Schaberg KB, Novoa RA, Wakelee HA, Kim J, Cheung C, Srinivas S and Kwong BY: Immunohistochemical analysis of lichenoid reactions in patients treated with anti-PD-L1 and anti-PD-1 therapy. J Cutan Pathol 43: 339-346, 2016.

9. Shi VJ, Rodic N, Gettinger S, Leventhal JS, Neckman JP, Girardi M, Bosenberg M and Choi JN: Clinical and histologic features of lichenoid mucocutaneous eruptions due to anti-programmed cell death 1 and anti-programmed cell death ligand 1 immunotherapy. JAMA Dermatol 152: 1128-1136, 2016.

10. James DG: A clinicopathological classification of granulomatous disorders. Postgrad Med J 76: 457-465, 2000.

11. Benn BS, Lombard CM and Krishna G: Nivolumab-induced granulomatous inflammation of the Pleura. J Thorac Oncol 12: e100-e101, 2017.

12. Danlos FX, Pagès $C$, Baroudjian $B$, Vercellino L, Battistella $M$, Mimoun M, Jebali M, Bagot M, Tazi A and Lebbé C: Nivolumab-induced sarcoid-like granulomatous reaction in a patient with advanced melanoma. Chest 149: e133-e136, 2016.

13. Wu J, Kwong BY, Martires KJ, Rieger KE, Chung WH, Iyer GV and Lacouture ME: Granuloma annulare associated with immune checkpoint inhibitors. J Eur Acad Dermatol Venereol 32: e124-e126, 2018

14. Hakeem A, Bhattacharyya I, Aljabri M, Bindakhil M, Pachigar K, Islam MN, Cohen DM and Fitzpatrick SG: Lichenoid reaction with granulomatous stomatitis: A retrospective histologic study of 47 patients. J Oral Pathol Med 48: 846-854, 2019.

15. Ferguson A, Golden S and Morrison L: New-onset oral lichen planus and granulomatous cheilitis in a 66-year-old woman. JAAD Case Rep 2: 177-180, 2016.

16. Gonzalez JG, Marcus MD and Cruz DJ: Giant cell lichenoid dermatitis. J Am Acad Dermatol 15: 87-92, 1986.

17. Magro CM and Crowson AN: Lichenoid and granulomatous dermatitis. Int J Dermatol 39: 126-133, 2000. 
18. Robinson CM, Oxley JD, Weir J and Eveson JW: Lichenoid and granulomatous stomatitis: An entity or a non-specific inflammatory process? J Oral Pathol Med 35: 262-267, 2006.

19. Hofmann L, Forschner A, Loquai C, Goldinger SM, Zimmer L, Ugurel S, Schmidgen MI, Gutzmer R, Utikal JS, Göppner D, et al: Cutaneous, gastrointestinal, hepatic, endocrine, and renal side-effects of anti-PD-1 therapy. Eur J Cancer 60: 190-209, 2016.

20. Sibaud V, Eid C, Belum VR, Combemale P, Barres B, Lamant L, Mourey L, Gomez-Roca C, Estilo CL, Motzer R, et al: Oral lichenoid reactions associated with anti-PD-1/PD-L1 therapies: Clinicopathological findings. J Eur Acad Dermatol Venereol 31: e464-e469, 2017

21. Diaz-Perez JA, Beveridge MG, Victor TA and Cibull TL: Granulomatous and lichenoid dermatitis after IgG4 anti-PD-1 monoclonal antibody therapy for advanced cancer. J Cutan Pathol 45: 434-438, 2018.
22. Khelifa-Hamdani E, Touati-Serraj M, Perriard J, Chavaz P, Saurat JH and Kaya G: Giant cell lichenoid dermatitis in a patient with baboon syndrome. J Cutan Pathol 35 (Suppl 1): S17-S19, 2008.

23. Errico A: Immunotherapy: PD-1-PD-L1 axis: Efficient checkpoint blockade against cancer. Nat Rev Clin Oncol 12: 63, 2015.

24. Villadolid $\mathbf{J}$ and Amin A: Immune checkpoint inhibitors in clinical practice: Update on management of immune-related toxicities. Transl Lung Cancer Res 4: 560-575, 2015.

25. Costa NL, Gonçalves JAM, de Lima SLG, de Arruda JAA, Miranda ACC, Mesquita RA, da Silveira ÉJD and Batista AC: Evaluation of PD-L1, PD-L2, PD-1 and cytotoxic immune response in oral lichen planus. Oral Diseases 26: 1246-1254, 2020.

cc) (i) $\odot$ This work is licensed under a Creative Commons

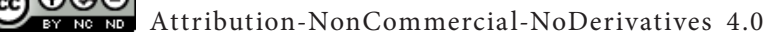
International (CC BY-NC-ND 4.0) License. 